\section{Gloria, Afrenta Y Pesar Del Protector Del Perú(1)}

\author{
Glory, Affront and Sorrow of the Protector of the \\ Peru
}

\section{Ricardo Pinto-Bazurco MendozA ${ }^{(2)}$}

\begin{abstract}
Resumen
El inexorable paso del tiempo avanza y con él se recrudecen los pesares y dolores propios de una vida que se apaga. Una intensa vida que estuvo inmersa en gloria pero que en su ocaso solo encontró, en los recuerdos de su propio autor, el único reconocimiento permanente que, lamentablemente, solo le otorgó la posteridad. Saludos y honores que, paulatinamente, se apagaron; situación que se manifestó a través de tres momentos claves expresados en elogios, pasquines y epístolas que nos permiten tener una clara idea de la relación que mantuvo el general José de San Martín y Matorras con una elite limeña que se encontró en vilo, económica y socialmente, como resultado de un plan político y una estrategia que no buscaron otra cosa que la libertad del Perú, y con ello sellar la independencia de Sudamérica. Esta relación se resume perfectamente en la gloria, afrenta y dolor del Protector del Perú.
\end{abstract}

Palabras claves: José de San Martín, independencia, Perú.

\section{Abstract}

The inexorable passage of time advances and, with it, intensifies the sorrows and pains of a life that goes out. An intense life that was immersed in glory but which in its twilight only found, in the memories of its own author, the only permanent recognition that, unfortunately, only granted him posterity. Greetings and

1 Este trabajo fue presentado en la mesa de "debate político" del VII Congreso "Hacia el Bicentenario de la Independencia" organizado por el Vicerrectorado de Investigación y Posgrado, la Facultad de Ciencias Sociales y la Escuela Académica Profesional de Historia de la Universidad Nacional Mayor de San Marcos, el 29 de noviembre del 2016.

2 Historiador. Universidad Nacional Mayor de San Marcos. Instituto Raúl Porras Barrenechea. Benemérita Sociedad Fundadores de la Independencia, Vencedores el 2 de Mayo 1866 y Defensores Calificados de la Patria.

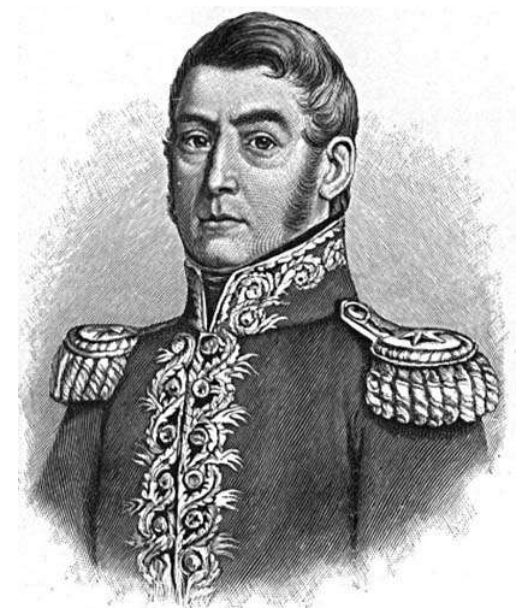

El Protector de El Perú, Capitán General D. José de San Martín y Matorras. Grabado inspirado en la litografía de Madou, Bruselas, 1828. Foto: Biblioteca personal

honors that gradually went out; situation that manifested itself through three key moments expressed in praise, pasquins and epistles that allow us to have a clear idea of the relationship that General José de San Martín y Matorras maintained with a Lima elite that found itself hanged, economic and socially, as a result of a political plan and a strategy that sought nothing but the freedom of Peru, and thereby sealing the independence of South America. This relationship is perfectly summed up in the glory, affront and sorrow of the Protector of Peru.

Keywords: José de San Martín, independence, Peru.

1 Médico internista. Facultad de Medicina, Universidad Peruana Cayetano Heredia.

2 Universidad de Berna, Suiza. 


\section{INTRODUCCIÓN}

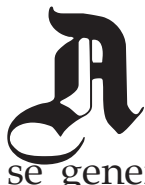

pocos de conmemorar el bicentenario de la proclamación de nuestra independencia varias interrogantes se generan por si solas: ¿El Perú ha logrado ser una nación integrada?, ¿La sociedad peruana ha logrado amalgamar su identidad cultural?, ¿El Perú está en el camino correcto del desarrollo en comparación a aquellos que ya cumplieron su bicentenario?, ¿El Perú ha logrado afianzarse estructuralmente para afrontar los retos del mañana?

Estas y otras preguntas resultan ante la natural incertidumbre que genera el derrotero de un país cuyo deber es buscarel gran norte de seruna nación justa y con igualdad de oportunidades como se pretendió hace casi doscientos años. Al recordar un viejo apotegma de José Martí que reza: "Lo pasado es la raíz de lo presente. Ha de saberse lo que fue, porque lo que fue está en lo que es", aplicado a nuestra realidad histórica, se puede notar que muchos de los aspectos que nos destacan o que nos caracterizan tienen una fuerte tradición de centenaria raigambre social que nos lleva a referirnos a los hechos y/o personajes, que tuvieron algún tipo de papel en nuestra historia, no de la manera más grata. Admonición que Jorge Basadre notó al estudiar la formación de la figura histórica de Ramón Castilla y que a su turno hiciera lo propio Raúl Porras Barrenechea al estudiar a Monteagudo y Sánchez Carrión.

En tal sentido los hechos acaecidos en torno a la figura del general José de San Martín y Matorras, el "Protector del Perú", se convierten en un caso emblemático de nuestra historia por ser el primer personaje político, del naciente nuevo régimen, que no escapó a las duras críticas de sus contemporáneos lo cual hizo mella en su persona ocasionando pesar y dolor en el cenit de su vida.
Los saludos y honores se fueron difuminando, hecho que se manifiesta a través de tres momentos clave expresados en elogios, pasquines y epístolas de la época que nos permiten tener una clara idea de la relación que mantuvo el general con el ámbito limeño ante la inesperada propuesta de un plan político y una estrategia que no buscaron otra cosa que la libertad del Perú y, con ello sellar, la independencia de Sudamérica ( $\mathrm{O}$ 'Phelan, 2008).

\section{Contexto Histórico}

El proceso de independencia en nuestro continente tuvo asidero, en su primera fase, en medio de la convocatoria a las Cortes Generales de España en Cádiz en 1810 y la posterior promulgación de la Constitución Española de 1812(3) (Guerra, 2016) cuyo talante liberal, en contraposición al poder monárquico, se fue decantando conforme la sociedad peruana, que mantuvo un sentimiento a favor de la separación de España (Carlos Contreras, Marina Zuloaga, 2016), asimilaba sus fundamentos cuyo norte era alcanzar la igualdad de los futuros ciudadanos bajo un nuevo orden político. Criterios que, paulatinamente, se dispersaron sobre cada miembro de la sociedad como una pandemia que se fue contagiando, pero que se encontraba subyugada ante la enérgica resistencia del virrey Fernando de Abascal. Empero la llegada del Ejército Libertador fue aquella esperada oportunidad que sirvió como caldo de cultivo para el desarrollo de las campañas militares entre 1820 y 1824.

3 Estableció la reunión e igualdad de los españoles de la metrópoli y las colonias y se declaró la independencia de España. Los diputados del Perú fueron: Dionisio Inca Yupanqui, Antonio Zuazo, José Lorenzo Bermúdez, Pedro García Coronel, Ramón Feliú, Vicente Morales Duárez, Blas Ostolaza, Francisco Salazar y José Antonio Navarrete. 


\section{GLORIA}

La figura paradigmática de José Francisco de San Martín y Matorras ${ }^{(4)}$, cuyo paso por el Perú dejó abigarradas opiniones, no gozó de los perpetuos elogios y enhorabuenas que en un primer momentolefueron inherentes a su figura de Libertador. Tal y como fueron los aplausos y vítores de gloria que recibió en el pomposo y retórico Elogio, el cual era reservado solo para los virreyes en su bienvenida a la Ciudad de los Reyes. Aquel día ${ }^{(5)}$, en el Salón General de la Universidad de San Marcos de Lima ${ }^{(6)}$ y bajo la atenta mirada del Protector, hizo uso de la palabra el doctor Justo Figuerola ${ }^{(7)}$ quien entre gongóricas hipérboles inició el panegírico con estas palabras:

"La Primera Escuela del Perú, enagenada de gozo al contemplar la independencia de esta América, consagra al Genio de la Libertad los homenajes mas sinceros, esperando que los mas dignos se tributen á V.E por la posteridad, cuando los talentos de la Patria hayan elevado a su vuelo á la altura á que no pueden tocar el dia los primeros sabios, que la condecoran é ilustran. Si, Excmo, Sr. Para esa época reserva la Universidad de San Marcos pagar á V.E esa deuda, que hoy solo se complace en publicar, para que los hijos de la nueva Atenas cubran de crédito que dejarán sus padres insoluto." (Elogio 1822:1)

4 Nació en Yapeyú (Argentina), el 25 de febrero de 1778. Sus padres fueron Juan de San Martín y Gregoria Matorras del Ser, ambos españoles. Se casó con María de los Remedios de Escalada con quien tuvo a su única hija; Mercedes Tomasa San Martín y Escalada.

5 Fue leído el 17 de enero de 1822.

6 La sede universitaria estuvo ubicaba frente de la Plaza del Estanque o de la Inquisición desde 1577, en lo que fue la Casa para jóvenes mestizas de San Juan de la Penitencia, hasta 1867 que es trasladada a lo que fuere la sede del Convictorio de San Carlos.

7 A la sazón, diputado segundo del Ilustre Colegio de Abogados, catedrático de Vísperas de Leyes, procurador general de la Universidad de San Marcos y notario mayor del Arzobispado de Lima.
Fue sin lugar a dudas uno de los gestos más importantes por el simbolismo que ello acarreaba puesto que la Universidad fue una de las instituciones más representativas del antiguo régimen que al suscribir el Acta de Independencia ratificaba su apoyo al nuevo orden político aun en ciernes. Cabe resaltar que José de San Martín fue el primer ilustre al que la universidad le otorgó el grado de Doctor Honoris Causa (Eguiguren, 1951). En otra parte del elogio leído tan histriónicamente, el emocionado narrador exclamó: "Viva la libertad, y el héroe por quien tiene vida" (Elogio 1822: 31), sin lugar a dudas la lisonja era la moneda corriente ante cualquier expresión que al Protector se refería.

El Elogio cierra su lauda recalcando los sentimientos que en pocos meses serían los primeros en estar ausentes:

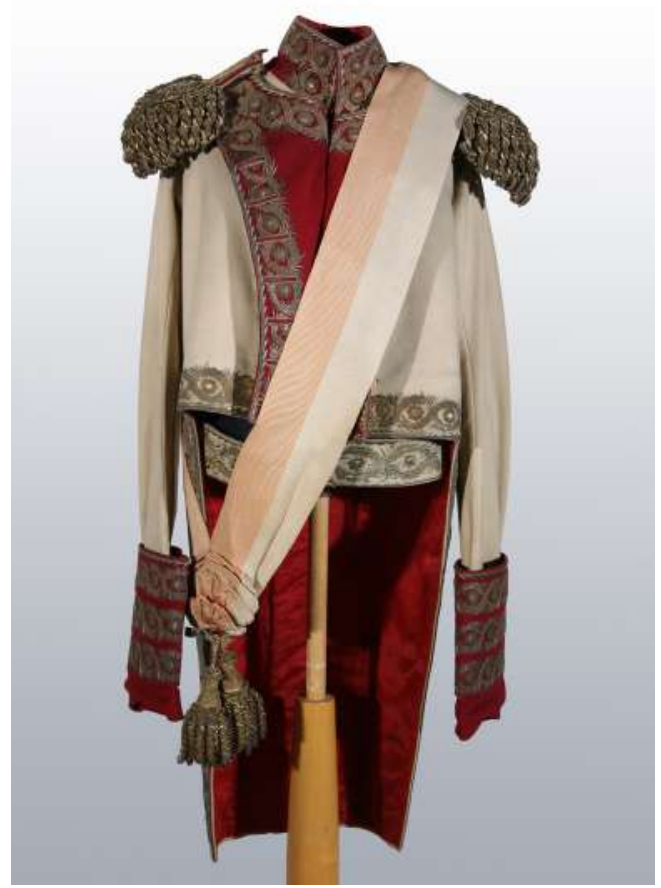

Uniforme de José de San Martín y Matorras con el grado de Capitán General (máximo grado militar por decreto del 30 de octubre de 1821). Sobre el mismo, la banda de Protector del Perú. Foto: Museo Histórico Nacional de Argentina. 
"Pueblos, repetid el nombre de vuestro Libertador, y encargad a las generaciones que os sucedan, que todas le consagren el tributo de la admiración, gratitud y ternura" (Elogio 1822: 39).

Meses después la gloria que envolvía al Protector fue dejando su espacio a la desazón, la zozobra y la desconfianza de la sociedad limeña. Es así que poco antes de emprender su retiro del Perú, San Martín, envió una carta a Simón Bolívar en la que expresó:

"He convocado al Congreso para presentar ante él mi renuncia y retirarme a la vida privada con la satisfacción de haber puesto a la causa de la libertad toda la honradez de mi espíritu y la convicción de mi patriotismo. Dios, los hombres y la historia juzgarán mis actos públicos." (8)

José de San Martín, quien ostentaba el título de generalísimo del ejército del Perú, enfrentó una marcada situación adversa producto de la grave colisión con un sector de la élite conservadora de la generalidad limeña. Por un lado, la clase dominante ya no encontraba legitimidad en el proyecto monárquico, ya que tal carecía de autenticidad por no provenir de un noble (O' Phelan Godoy 2010:68).

La construcción mental de la elite limeña determinaba que sus pares eran los únicos quienes podrían ostentar cualquier tipo de cargo en la administración y con mayor razón plantear cualquier tipo de nuevo régimen o sistema político. Si bien es cierto que San Martín, con la intención de respaldar su "monarquía constitucional", desarrolló una serie de mecanismos concesionarios y de filiación como la creación de la Orden del Sol

8 Esa carta fue enviada el 10 de septiembre de 1822, doce días antes de la instalación de la Asamblea Constituyente de Lima.

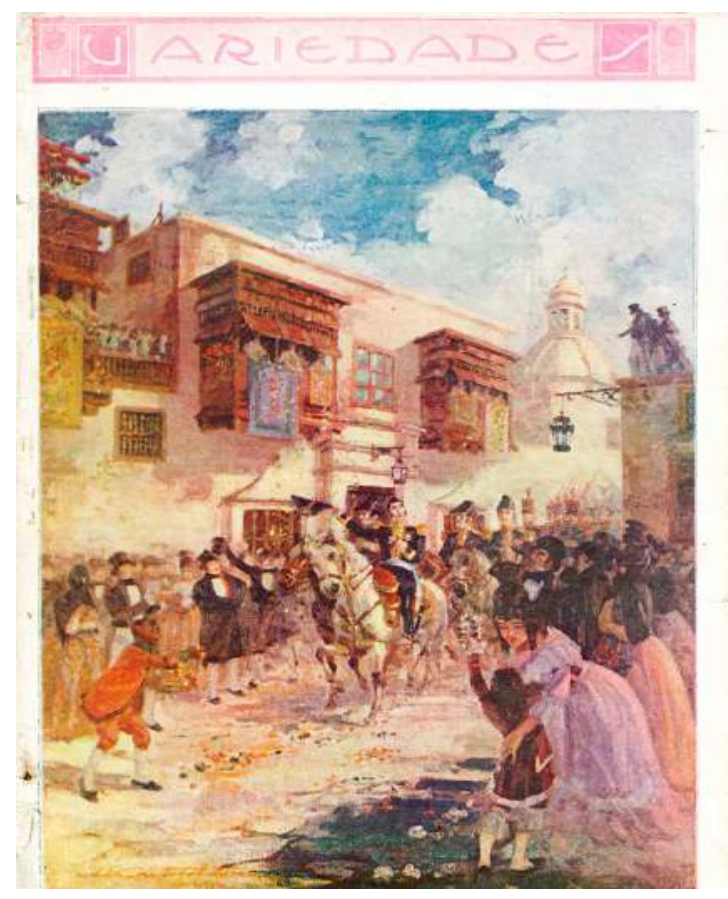

Carátula de la revista "Variedades". Edición especial por Fiestas Patrias. Julio de 1921. Aparece en ella "San Martín ingresando a Lima”, óleo de José Alcántara la Torre (18931978). Foto: Biblioteca IRPB-UNMSM.

del Perú( ${ }^{(9)}$ (ISMP 1971,13) y la adhesión de connotados titulados y aristócratas como José Bernardo de Tagle y Portocarrero, IV marqués de Torre Tagle y, el no menos importante, José Mariano de la Cruz de la Riva-Agüero y Sánchez Boquete por citar algunos ejemplos. Empero la relación entre la élite y San Martín se corroyó no solo por lo antes mencionado sino por una serie de factores cuyo eje principal radicó en la postura y acción anti-hispánica que preconizaba su ministro Bernardo José de Monteagudo quien a su turno refirió: "en el curso de unos meses esperaba desterrar del Perú a todos los tiranos y pillos españoles" (Mathison 1973: 317).

9 Fue establecida mediante decreto el 8 de octubre de 1821. Estuvo dividida en tres clases: Fundadores, Beneméritos y Asociados a la Orden del Sol. La Orden fue suprimida el 9 de marzo de 1825 para luego ser reestablecida en 1921, por el presidente Augusto B. Leguía, cuya vigencia continua hasta nuestros días. 
Por otro lado, las falsas expectativas que se generaron en los sectores populares, básicamente entre campesinos indígenas y negros esclavos, se tradujeron en hechos tangibles como la paradójica exoneración del tributo indígena a cambio de enrolarse en las milicias lo cual no se cumplió permanentemente debido a que disminuía la principal mano de obra en la producción agrícola. Asimismo, la no manumisión general ya que la mano de obra de los esclavos negros resultaba, cuantitativa y cualitativamente, importante para los haciendas y hogares en la ciudad. Estas medidas solo demostraron que los indígenas y esclavos no fueron parte preponderante del gran proyecto cuya inclusión en el nuevo régimen estuvo lejos de sus expectativas debido a que las medidas que los "beneficiarían" tuvieron un carácter más coyuntural que estructural. $\left(\mathrm{O}^{\prime}\right.$ Phelan Godoy 2010:86).

En medio de este desencantado ambiente en el que "no se sentía el espíritu de nacionalidad y el entusiasmo patriótico" (Mathison 1973: 250) el otrora Protector se retiraba del Perú, el 21 de septiembre de 1822. Luego del largo periplo hacia el sur llegó a Chile visiblemente enfermo y deprimido. Postrado por la enfermedad y lastimado por la ingratitud pasó sesenta y seis días en cama hospedado por un amigo en una quinta ubicada por los alrededores de Santiago a inmediaciones del famoso llano de Maipo. Apenas convaleciente se le presentó uno de sus antiguos compañeros pidiéndole una "habilitación" creyéndolo millonario según se decía. Con tal motivo escribió con pulso trémulo y desgarradora ironía a su amigo Bernardo O'Higgins, peregrino como él: “Estoy viviendo de prestado. Es bien singular lo que me sucede, y sin duda pasaráa usted lo mismo, es decir, están persuadidos de que hemos robado a troche $y$ moche. jAh, picaros! ¡Si supieran nuestra situación, algo más tendrían que admirarnos;" (Mitre: 1878).
La indignación de San Martín fue, sin duda, muy marcada. Si bien es cierto que el gobierno del Perú al tener noticias de la precaria condición del Protector envió dos mil pesos de sus sueldos, lo cual le sirvió para continuar su camino hacia Mendoza en 1823, fue inevitable que la pena y desdicha se incrustaran profundamente en los sentimientos de José de San Martín que pasó a tener una humilde vida de chacarero.

Ahora los comentarios hacia él se tornarían en injurias, viles acusaciones y colosales ingratitudes.

\section{Afrenta}

Para 1823, en Lima, los panfletos hacían escarnio de la menoscabada figura del Protector a través de dichos y desdichos tal y como se puede constatar mediante el análisis de la tribuna de uno de los más conspicuos hombres que proponían y promovían el nuevo orden republicano, José Faustino Sánchez Carrión. En la célebre Abeja Republicana, en medio de la creciente y desmedida ingratitud hacia San Martín y todo lo que al él se relacionaba, se publicó en el número cinco de la mencionada publicación un artículo en detrimento de San Martín y los jefes de su ejército lo cual no hacía más que aumentar el desaguisado que buscaba deslegitimar las acciones emprendidas por ellos en su hora. Esto a pocos días del ingreso de Bolívar a la ahora llamada Ciudad de los Libres. Ante tal afrenta, un grupo que se autodenominó "Los Amigos de la Libertad" dedicaron un desagravio que buscó frenar las injustas manifestaciones de las que era objeto San Martín y su ejército. En esta particular impugnación "Los amigos de la Libertad" recordaron, dura y puntualmente, el carácter endeble que los limeños tuvieron ante el sistema del antiguo régimen colonial. Escribieron: "en esta ciudad no se oyó sino el 
lijero susurro del descontento, y su agitación fue semejante a la que riza blandamente la superficie de las aguas" (Abeja Republicana 1823: 2).

Por su parte los detractores de San Martín sostenían que a su retiro dejó un permanente estado de anarquía al haber cesado en sus funciones a las autoridades del Estado, además, que las finanzas del país estaban destruidas entre otras muchas complicaciones. Se acusaba que muchas de las medidas tomadas por San Martín fueron hechas sin la previsión del caso generando un marcado estado de inestabilidad sobre todo en los sectores dominantes de la sociedad que a la larga fueron quienes le quitaron el apoyo y por ende el reconocimiento permanente a su gesta (O'Phelan, 2008).

\section{Pesar}

El inexorable paso del tiempo avanza y con él, quedaron atrás las recompensas y honores; y al mismo tiempo se recrudecieron los pesares y dolores propios de una vida que se apaga. Una intensa vida que estuvo inmersa en gloria, pero que en su ocaso solo encontró en los recuerdos de su propio autor, el único reconocimiento permanente que, lamentablemente, solo le otorgó la posteridad.

Como evidencia de ese olvido del que, el Protector, se sentía preso se encuentra una epístola cuyo testimonio vívido demuestra el real sentimiento del general José de San Martín y Matorras. Esta fue remitida, el 11 de setiembre de 1848 desde su autoexilio en la que también sería su morada final la localidad francesa de Boulogne-sur-Mer ${ }^{(10)}$, a quien, a la sazón, era el presidente constitucional de la República del Perú, en respuesta a una carta que el mismo

10 Localidad del norte de Francia, en el Departamento del Paso de Calais. Se encuentra junto al Canal de la Mancha a orillas del encuentro entre el Océano Atlántico y el Mar del Norte.

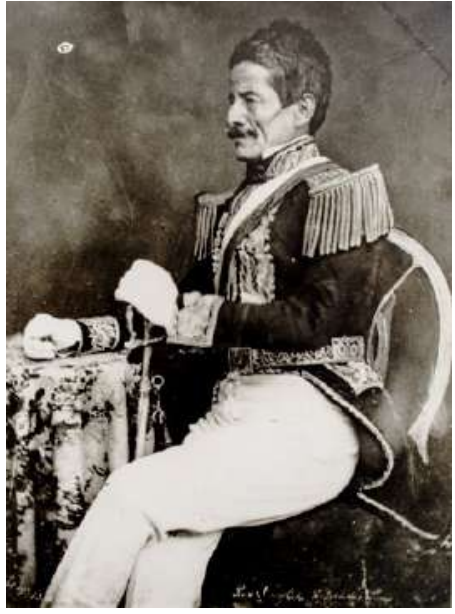

Presidente del Perú Gran D. Ramón Castilla y Marquesado durante su primer gobierno constitucional 1845-1851. Foto: Internet

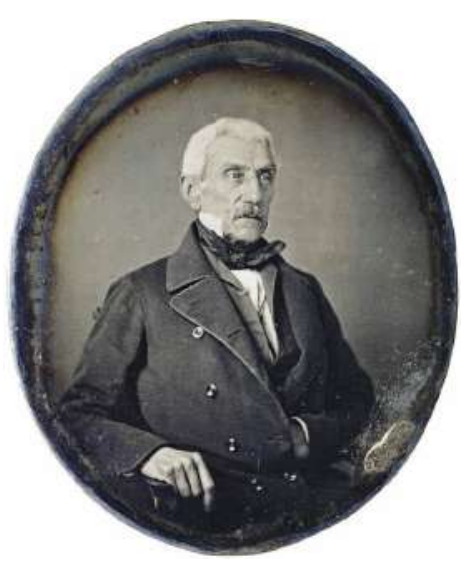

Generalísimo de las armas, Fundador de la Independencia y Protector de la Libertad del Perú D. José de San Martín y Matorras a los setenta años (Daguerrotipo. París, 1848).

general Ramón Castilla y Marquesado (18451851) ${ }^{(11)}$ le enviara desde Lima, el 13 de mayo del mismo año. A la luz de este documento se aprecia la cercana relación epistolar entre el Protector y el mariscal, quien en una carta previa da cuenta de algunos detalles de su carrera militar a la que José de San Martín, con

11 Para esos años, Castilla, se encontraba al final de su primer gobierno como Presidente Constitucional de la República del Perú, experiencia que sumaba a la presidencia de la Suprema Junta de Gobierno Provisorio en reemplazo, por muerte, del general Domingo Nieto (1844). 
setenta años, responde con una reseña de los principales hechos de su vida que empieza de la siguiente manera: "Usted me hace una exposición de su carrera militar bien interesante; a mi turno permítame le dé un extracto de la mía". (Revista Peruana 1879: 40-43).

El pesar y la congoja embargaban al ya viejo Protector sus recuerdos plasmados en las epístolas así lo demuestran: " a la edad avanzada de 71 años, una salud enteramente arruinada $y$ casi ciego con la enfermedad de cataratas, esperaba, aunque contra todos mis deseos, terminar en este país una vida achacosa" (Revista Peruana 1879: 40-43). Con el ánimo mustio alejado de su patria por un "auto ostracismo", como el mismo lo denominó, no dudó en compartir sus sentimientos con Ramón Castilla.

En otro pasaje de la epístola mencionó lo relacionado al tema monetario y la deuda que el Estado peruano le tuvo y que hasta ese entonces no parecía cancelada:

"Un millón de gracias por sus francos ofrecimientos; yo los creo tanto más sinceros cuanto son hechos a un hombre que, por su edad y achaques, es de una entera nulidad; yo los acepto para una sola cosa, a saber, rogar a usted que los alcances que resultan de los ajustes de mi pensión hechos por esas oficinas puedan, si es de justicia, ser reconocidos por el Estado; pero con la precisa circunstancia de que nada será satisfecho hasta después de mi fallecimiento, en que mis hijos encuentren este cuerpo de reserva para su existencia." (Revista Peruana 1879)

Es notoria la respuesta de gratitud ante el ofrecimiento que le hiciera Castilla del pago de sus sueldos correspondientes, En otro párrafo, San Martín, revela los sentimientos que le embargaron luego de su retiro de Lima lo cual demuestra que gozó de un carácter desprendido y generoso:
"Si algún servicio tiene que agradecerme la América, es el de mi retirada de Lima, paso que no sólo comprometía mi honor y reputación, sino que me era tanto más sensible, cuanto que conocía que con las fuerzas reunidas de Colombia, la guerra de la Independencia hubiera sido terminada en todo el año 23. Pero este costoso sacrificio, y el no pequeño de tener que guardar un silencio absoluto (tan necesario en aquellas circunstancias), de los motivos que me obligaron a dar este paso, son esfuerzos que usted podrá calcular y que no está al alcance de todos el poderlos apreciar." (Revista Peruana 1879).

La relación epistolar duró un tiempo más hasta que en una carta escrita por Mariano Balcarce, esposo de Mercedes Tomasa San Martín y Escalada, hija del libertador don José de San Martín, remitida al presidente de la República Ramón Castilla, el 14 de setiembre de 1850, se anunciaba la muerte del Protector que había acaecido el 17 de agosto de 1850 producto de una hipertrofia cardiaca. Además, se mencionaba en dicha carta, el último gesto de desprendimiento que el Protector tuvo hacia el Perú, el cual hizo agregar a su testamento, fue la devolución del estandarte que Francisco Pizarro usó para conquistar el Imperio de los Incas. Tal vez luego de reflexionarlo, la sutileza del presidente Castilla tuvo éxito ya que en una carta que el Mariscal envía a San Martín se menciona:

"En mi carta del 13 me tomé la libertad de llamar la atención de U. sobre otro diverso e importante objeto: hablo del estandarte de Pizarro y del expediente de Santa Rosa de Lima, que cuando $U$. se retiró del Perú llevo consigo, como recompensa más distinguida a los servicios que U. había prestado a esta República.

Suponiendo, como debo suponer, que U. quiso poseer aquellos trofeos por un tiempo determinado, a los más durante sus dias, que celebraría fuesen perdurables; y tomándose la 
confianza de ser intérprete de su voluntad, que siempre juzgué hon rosa y amigable del Perú, creo que $U$. recibirá con agrado mi indicación y seservirádecirme suopinión y última disposición respecto al estandarte de Pizarro y expediente de Santa Rosa que creo deben volver a esta República, si no antes, inmediatamente después de los dias de U." (Castilla 1849)

Enterado del deceso de San Martín el presidente Castilla ordenó, a través de un decreto supremo dado el 7 de noviembre de 1850, disponer que se preparen: "en las capitales de los departamentos y provincias litorales de la República se harán exequias por el alma del finado Jeneralísimo D. José de San Martín con la asistencia de autoridades y corporaciones" (Colección de leyes y decretos 1850: 152). Además, en el mismo decreto supremo, se estableció que en el centro de la plazuela llamada "Siete de setiembre" se erija una columna de veinte pies de altura, sobre la cual se colocará la estatua de San Martín. Lamentablemente, este gesto no se concretó de inmediato pero marcó el inicio del eterno y permanente reconocimiento a la gesta libertaria que el Protector realizó en nuestro territorio.

\section{Conclusión}

A la luz de la historia y sobre las ideas sustanciales expuestas se puede colegir que la construcción de la figura histórica del Protector del Perú se sostiene fundamentalmente sobre dos pilares historicistas:

Por un lado, la representación o sistema de representación, mediante la cual un individuo o grupo social se apropia de su entorno cultural, de una eventual proyección, de sí mismo y del otro le permite determinar diferentes aspectos en las relaciones sociales. En tal sentido fueron los afanes y pesares que atravesó el Protector, José de San Martín, elementos que lo convierten en un paradigma para la sociedad debido a lo trascendente de su protagonismo en el desarrollo de la etapa temprana del nuevo régimen en la historia del Perú. Hecho que generó antagónicos sentimientos de crítica y adhesión entre sus contemporáneos; expresiones de las que no fue ajeno. Por otro lado, y como complemento, la apreciación o sistema de apreciación que surge como consecuencia de la representación, que no hace más que ordenarla, determina las modalidades de observación del entorno y de sí mismo, del individuo o grupo social. Ergo, la "aptitud criolla para solazarse en el asesinato de las reputaciones" (Basadre, 1954) juega un papel fundamental debido a que la crítica desgarradora permite construir una estructura sobre la que se posa la figura histórica y así contrapesar los aspectos positivos y negativos del personaje en cuestión.

En suma, ambos conceptos conforman un sistema que organiza los vínculos afectivos de la generalidad con un individuo (la figura histórica de El Protector), lo cual determina un patrón de comportamiento social a favor o en contra. De esta manera no solo se convierte en un medio sino también, y al mismo tiempo, en un fin en sí mismo cuyo trasfondo principal es socio-histórico.

Finalmente, como refiriera el maestro Porras: "no hay patria sin historia y que las primeras expresiones de esta son los himnos de triunfo y los cantares guerreros y que, por ende, a despecho de filósofos socializantes, la historia tiene siempre una inarrancable entraña épica", tal y como lo vivó, el Protector del Perú.

\section{REFERENCIAS BibLIOGRÁficAs}

1. Basadre, J. (1954). La Formación de la Figura Histórica de Ramón Castilla. Mercurio Peruano. 1954; XXXV(331), 721-751.

2. Carlos Contreras, Marina Zuloaga. Historia Minima del Perú. 
Madrid: Turner Publicaciones; 2016.

3. Castilla, R. Decreto 161. Se manda hacer ezequias fúnebre, Jeneralísimo San Martín y que vistan de luto los empleados civiles y militares de la República. Lima; 1850.

4. Eguiguren, L. A. Diccionario histórico-cronológico de la Universidad Real y Pontificia de San Marcos y sus Colegios. Lima: Impr. Torres Aguirre; 1951.

5. Guerra, M. (Ed.). Cronología de la Independencia del Perú (Guarrea, Margarita ed.). Lima: IRA- PUCP; 2016.

6. Hampe, T. Miguel Grau, proyagonista político. Piura: Caramanduca Editores; 2013

7. Loayza, A. (Ed.). La independencia peruana como representación. Historiografía, conmemoración y escultura peruana. Lima: IEP; 2016.

8. Mathinson, G. Residencia en Lima entre abril y mayo de 1822. En En Relación de viajeros. Lima: Colección Documental de la Independencia; 1973.

9. O'Phelan, S. El general don José de San Martín y su paso por el Perú. Lima: Editorial del Congreso del Perú; 2008.

10. Perú, I. S. La Orden del Sol del Perú. Lima: Talleres gráficos PL Villanueva S.A.; 1971.

11. Porras. José Sánchez Carrión, el tribuno de la República Peruana. Conferencia sustentada en el Centro de Estudios Históricos Militares. Lima: Talleres de Artes Gráficas “Tipografía Peruana” S.A.; 1953: 31.

12. Porras, R. Elogio y vejamen de la Reùblica (Monteagudo y Sánchez Carrión). En J. Puccinelli, Antología de Raúl Porras. Lima: CEPREFDIM - UNMSM; 2011: 115-118.

13. San Martín. El hombre y el miedo. Ensayo de interpretación de una conducta. (1965). Buenos Aires: Ministerio de Educación y Justicia; 1965.

14. Sanmartiniano, I. N. Renunciamientos del capitán general don José de San Martín. Buenos Aires: INS-Argentina; 1969.

15. Sanmartiniano, I. N. Cartas del gran mariscal don Ramón Castilla presidente del República del Perú al fundador de la independencia y protector de la libertad del Perú, generalísimo de las armas don José de San Martín. Buenos Aires: INSArgentina; 1973.

\section{FUENTES IMPRESAS}

\section{En John Carter Brown Library-Peru Collection:}

- 1822. Elogio del excelentisimo señor don Jose de San Martin y Matorras: protector del Peru, generalisimo de las fuerzas de mar y tierra, institutor de la Órden del Sol, gran oficial de la legion de merito de Chile, y capitan general de sus exercitos, que en su publico recibimiento en la Universidad de San Marcos de Lima el dia 17 de enero del presente año, [2], 39, [5] p. $20 \mathrm{~cm}$. (8vo), Lima.

- 1823. Impugnacion al articulo inserto contra el fundador de la libertad del Peru, y los jefes de su ejercito en el numero 5 de la Abeja republicana. Escrita y dedicada a estos por los Amigos de la libertad, [4], 20 p. c29 cm. (fol.), Lima.

- 1848. Carta del Presidente del Perú, Gran Mariscal don Ramón Castilla al Generalísimo de las armas peruanas, don José de San Martín, noviembre 13 de 1848, Lima.

- 1849. Carta del Presidente del Perú, Gran Mariscal don Ramón Castilla al General don José de San Martín, mayo 26 de 1849, Lima.

- 1850. Decreto $N^{\circ} 161$ Se manda hacer ezequias fúnebre, Jenereralísimo San Martín y que vistan de luto los empleados civiles y militares de la República dado por Ramón Castilla. En Colección de Leyes, Decretos y Órdenes publicados en el Perú desde si Independencia (1848-1849) Lima: Imprenta de la Colección por Mariano Osorio (1853).

- 1879 Carta del libertador Don José de San Martín al presidente del Perú, general Don Ramón Castilla, 11 de septiembre de 1848, Revista Peruana, Lima. 